\title{
Nouvelles visions pour l'éducation nationale en Slovaquie
}

\section{Anna Butašová et Arnaud Segretain}

\section{OpenEdition}

12 Journals

Édition électronique

URL : https://journals.openedition.org/ries/5445

DOI : $10.4000 /$ ries. 5445

ISSN : 2261-4265

Éditeur

France Education international

\section{Édition imprimée}

Date de publication : 1 septembre 2016

Pagination : 19-22

ISBN : 978-2-85420-611-1

ISSN : $1254-4590$

Référence électronique

Anna Butašová et Arnaud Segretain, « Nouvelles visions pour l'éducation nationale en Slovaquie », Revue internationale d'éducation de Sèvres [En ligne], 72 I septembre 2016, mis en ligne le 01 septembre 2018, consulté le 05 juillet 2021. URL : http://journals.openedition.org/ries/5445; DOI : https://doi.org/ $10.4000 /$ ries. 5445

Ce document a été généré automatiquement le 5 juillet 2021.

(c) Tous droits réservés 


\title{
Nouvelles visions pour l'éducation nationale en Slovaquie
}

\author{
Anna Butašová et Arnaud Segretain
}

1 Il existe, dans le système de l'éducation nationale slovaque comme dans d'autres systèmes éducatifs d'Europe centrale, de véritables efforts, depuis le début des années 1990, pour trouver une voie optimale vers la modernisation. Des changements rapides, souvent intuitifs, n'ont pas été véritablement fructueux pour l'éducation nationale slovaque. Alors que, jusque dans les années 1990, les résultats des comparaisons possibles de l'époque indiquaient que le système éducatif slovaque était en général plutôt satisfaisant, nous sommes aujourd'hui, après les efforts de réforme, paradoxalement confrontés à des échecs plutôt qu'à des succès (comme l'indiquent les mesures internationales des performances des systèmes éducatifs).

2 Ce constat d'échec est régulièrement lié à la problématique du manque de financement à tous les degrés et à tous les niveaux du système éducatif slovaque. Certes, ce problème est sérieux et, dans certains domaines, la question du statut de l'enseignant est vraiment critique, comme le montre l'étude de la Commission européenne (2013) qui porte sur les mesures pour améliorer du métier d'enseignant en Europe, à la suite de l'enquête internationale Talis 2013. Mais le financement n'est pas la cause de tous les échecs constatés. Un des problèmes majeurs est la quasi non-existence de standards pour la profession. Les concevoir améliorerait certainement la formation initiale des personnels pédagogiques et spécialisés et augmenterait sans aucun doute la qualité du processus éducatif de formation.

3 La réforme la plus importante qu'a connue le système éducatif slovaque a eu lieu en 2008. Il faut cependant mentionner que le système n'est passé, depuis 1989, par aucun audit concernant ses besoins, ses points faibles et ses potentialités. Les années 1990 ont été caractérisées par des tentatives de conception de stratégies nationales, qui, étant donné le manque d'analyses en termes de contenu ou de financement, n'ont atteint leurs objectifs que partiellement et les effets attendus ont été absents. L'on a aussi manqué d'analyses comparatives avec des systèmes éducatifs efficients, qui auraient pu permettre d'éviter un certain nombre d'erreurs. Ces erreurs étaient la conséquence de 
l'abandon de la recherche pédagogique et des sciences de l'éducation en général au nom d'une certaine orientation politique avant 1989. Il a donc manqué toute une génération de scientifiques et de chercheurs dans ce domaine, qui auraient pu consacrer leurs travaux à la modernisation du système éducatif. L'illusion d'une école de qualité datant du socialisme tchécoslovaque a commencé à se briser avec l'introduction par l'OCDE de mesures internationales des performances des systèmes éducatifs, au sein desquelles la Slovaquie n'a cessé d'occuper des places de plus en plus plus basses.

4 En 2008 fut adoptée une grande réforme éducative par la promulgation de la loi dite «sur l'école » $n^{\circ} 245 / 2008$. La loi tentait de définir les principes du système éducatif élémentaire et secondaire... mais à nouveau, elle ne fut pas suivie d'une réforme des contenus de la formation universitaire des enseignants et rien ne fut programmé non plus concernant le financement de cette réforme pourtant votée, promulguée et appliquée, laissant ainsi le travail à moitié fait. Cette réforme de 2008 est parfois aussi nommée " réforme de choc ", car l'aspect diachronique ne fut concrètement pas pris en compte. La loi décrète un ralentissement de la centralisation alors qu'elle ne fait que la renforcer. Le curriculum, appelé en Slovaquie " programme d'enseignement », est créé sur deux niveaux : d'abord comme « Programme d'enseignement national » et publié en tant que tel par l'État, puis au niveau de l'école sous l'appellation «Programme d'enseignement de l'école». Les contenus des programmes d'enseignement prévoient l'acquisition des compétences clés et décrivent les standards de contenus et de performance à acquérir par les apprenants. Dans le cas de la réforme de 2008, l'État a mené une expérience dont les conséquences marqueront les futures générations de chercheurs, d'enseignants, de parents et certainement aussi d'élèves, qui en ont fait les frais. Comme la loi renforce les organes centraux de décision, les changements politiques se reflètent très visiblement et chaque changement politique apporte ses changements curriculaires. Les macro-indicateurs généraux tels que le « Monitor 9 » (évaluation des élèves en fin de $9^{e}$ année de l'école slovaque), les standards d'enseignement, l'approche par compétences clés, l'orientation vers la performance sont autant d'indicateurs utiles à l'état pour présenter l'état de l'éducation nationale. L'inspection s'intéresse aussi à ces macro-indicateurs. L'État contribue de façon essentielle au développement de ces macro-indicateurs en introduisant les technologies de l'information et de la communication et en incitant de façon indirecte les écoles à continuellement améliorer leurs équipements. L'essence même de l'institution scolaire - l'enseignement, l'éducation et le développement de relations interpersonnelles disparaît non seulement du point de vue de l'État mais aussi des parents, des enseignants et du grand public. Ce sont des préoccupations telles que la place de l'école à l'échelle nationale, la place de la Slovaquie dans les classements internationaux, les débouchés pour les anciens élèves, le marché du travail qui ont le plus d'échos actuellement. Il s'agit là d'une tendance mondiale, plus ou moins prononcée selon les pays.

5 En 2013 a commencé, sous la pression populaire mais aussi suite à la publication d'un rapport sur l'école en Slovaquie (Ministère de l'éducation, 2013), une discussion publique qui portait principalement sur le contenu des programmes d'enseignement nationaux créés en 2008, en vue de les modifier. Les changements demandés faisaient référence à quelques manques essentiels dans les contenus, qui privilégiaient principalement les savoirs encyclopédiques et laissaient peu de place au développement des savoir-être de l'élève ou à la capacité d'utiliser les connaissances 
acquises dans la vie pratique, etc. L'accent fut porté également sur la question de la contribution de l'État au développement de l'autonomie de l'école. Dans quelle mesure l'école doit-elle être autonome, en ce qui concerne la scolarité obligatoire, et comment définir le droit de l'enfant à une éducation de qualité ? Toutes ces idées ont conduit en 2014 à des «programmes d'enseignement nationaux innovés » qui sont entrés en vigueur au $1^{\mathrm{er}}$ septembre 2015.

6 Il semble que la composante la plus moderne des curricula scolaires slovaques soit celle du domaine " langue et communication ", au sein duquel les langues étrangères sont à la pointe de la recherche actuelle. L'enseignement des langues étrangères est fondé sur les niveaux du Cadre européen commun de référence pour les langues (CECRL). Cependant, l'enseignement de la première langue étrangère (l'anglais) est devenu obligatoire dès la troisième année de la scolarité obligatoire. Jusqu'en 2011, les écoles pouvaient choisir la première langue étrangère qu'elles proposaient aux enfants et aux parents. L'anglais est devenu obligatoire surtout sous la pression des parents d'élèves, bien que la langue anglaise soit enseignée par un nombre important d'enseignants non qualifiés. À partir de la $6^{e}$ année, l'élève peut choisir une autre langue étrangère ; au lycée, deux langues étrangères sont obligatoires. Le baccalauréat slovaque (maturita) prévoit pour tous les bacheliers une épreuve obligatoire d'au moins une langue étrangère au niveau B2 du CECRL.

7 À l'automne 2015, dans le cadre d'un projet ${ }^{1}$ de développement des compétences professionnelles des enseignants des écoles maternelles, primaires et secondaires dans le contexte de l'augmentation de l'efficacité de la réforme du système d'enseignement, une recherche a montré, sur un échantillon d'enseignants stratifié, quels étaient les points sensibles de la réforme. Plus de 1600 enseignants d'écoles maternelles, primaires et secondaires ont participé à ce projet de recherche. L'enseignant, personne-clé d'un curriculum à deux niveaux, en est aussi l'auteur.

8 Selon $81 \%$ des enseignants interrogés, aucun changement important ne s'est réellement produit dans le secteur éducatif slovaque. $73 \%$ d'entre eux pensent que les réformes éducatives slovaques ne sont pas comparables à celles qui ont eu lieu dans le monde et $77 \%$ d'entre eux ont l'impression que l'école slovaque ne s'améliore pas. Malgré une position très négative vis-à-vis des réformes menées dans l'éducation, $59 \%$ des enseignants pensent que les réformes doivent continuer. Les enseignants des écoles maternelles et des lycées, respectivement $70 \%$ et $63 \%$, ont une position plus positive. Au niveau des écoles primaires, une forte polarisation des enseignants existe, avec $52 \%$ d'entre eux ressentant le besoin de changement, et pas les autres.

9 Ces développements récents dans le domaine éducatif indiquent que la situation en Slovaquie est grave. Savoir comment la résoudre reste une question ouverte. La plupart des spécialistes pensent qu'il est primordial d'investir dans la stabilisation du corps enseignant, de chercher à améliorer leur situation financière et, ce faisant, leur statut dans la société. Le deuxième point crucial concerne la formation initiale et continue des enseignants, au sein de laquelle il est nécessaire d'accroître leur autonomie et leur sentiment de copaternité du contenu des enseignements. Il est en outre indispensable d'œuvrer pour faire évoluer une autre perception, historique en Slovaquie : celle que la profession d'enseignant est dirigée « d'en-haut ».

10 Enfin dernier élément, mais non des moindres, il est nécessaire de se pencher sur le sujet et l'objet de l'éducation - l'enfant. Respecter ses droits mais aussi lui rappeler ses devoirs envers la société. 


\section{BIBLIOGRAPHIE}

EUROPEAN COMMISSION (2013): Study on Policy Measures to improve the Attractiveness of the Teaching Profession in Europe, final report, vol. 1, 84 p. [en ligne] DOI 10.2766/40827 ; vol. 2, 400 p. [en ligne] DOI 10.2766/41166, Luxembourg : Publications Office of the European Union.

MINISTÈRE DE L'ÉDUCATION DE SLOVAQUIE (2013) : Správa o stave školstva na Slovensku (Rapport sur l'état de l'école en Slovaquie), Bratislava [en ligne] [https://goo.gl/0AVO1b]

MINISTÈRE DE L'ÉDUCATION DE SLOVAQUIE (2008) : Štátny vzdelávací program 2008 (Programme d'enseignement national 2008) [en ligne] [http://goo.gl/lzOgGK]

OCDE (2014) : Guide TALIS 2013 à l'intention des enseignants : Enquête internationale sur l'enseignement et l'apprentissage, Paris : OCDE, DOI : http://dx.doi.org/10.1787/9789264216143-fr.

PORUBSKÝ Š., KOSOVA B., DOUSKOVA A. et al. (2014) : Škola a kurikulum (l'école et le curriculum), Université Matej Bel, Banská Bystrica.

ŠTÁTNY PEDAGOGICKÝ ÚSTAV (Institut pédagogique d'État) (2015) : Inovovaný štátny vzdelávací program 2015 (Programme d'enseignement national innové, 2015) [http://www.statpedu.sk/ clanky/inovovany-statny-vzdelavaci-program]

\section{NOTES}

1. Projet FSE, Institut national pédagogique, 2016.

\section{INDEX}

Mots-clés : programme d'enseignement, réforme de l'enseignement, système scolaire, curriculum

Palabras claves : programa de enseñanza, reforma de la educación, sistema escolar, curriculum Keywords : instructional programmes, educational reform, school system, curriculum Index géographique : Slovaquie

\section{AUTEURS}

\section{ANNA BUTAŠOVÁ}

Anna Butašová est directrice du département pour la communication plurilingue et interculturelle à l'Université Comenius de Bratislava (Slovaquie). Elle est présidente de la Commission nationale pour les langues étrangères auprès de l'Institut national de pédagogie et chercheuse en pédagogie à l'Institut national de pédagogie. Courriel : annabutasova@gmail.com 


\section{ARNAUD SEGRETAIN}

Arnaud Segretain est chercheur à l'Institut national de pédagogie de Bratislava (Slovaquie). Responsable de l'enseignement du français en Slovaquie, il enseigne également à l'Institut français à Bratislava. 